\title{
Exact non-Markovian master equation for a driven damped two-level system
}

\author{
H. Z. Shen ${ }^{1}$, M. Qin ${ }^{1}$, Xiao-Ming Xiu ${ }^{1,2}$, and X. X. Yi ${ }^{3}{ }^{*}$ \\ ${ }^{1}$ School of Physics and Optoelectronic Technology \\ Dalian University of Technology, Dalian 116024 China \\ ${ }^{2}$ Department of Physics, College of Mathematics and \\ Physics, Bohai University, Jinzhou 121013, China \\ 3 Center for Quantum Sciences and School of Physics, \\ Northeast Normal University, Changchun 130024, China
}

(Dated: July 1, 2014)

\begin{abstract}
Driven two-level system is a useful model to describe many quantum objects, particularly in quantum information processing. However, the exact master equation for such a system is barely explored. Making use of the Feynman-Vernon influence functional theory, we derive an exact nonMarkovian master equation for the driven two-level system and show the lost feature in the perturbative treatment for this system. The perturbative treatment leads to the time-convolutionless (TCL) and the Nakajima-Zwanzig (NZ) master equations. So to this end, we derive the time-convolutionless (TCL) and the Nakajima-Zwanzig (NZ) master equations for the system and compare the dynamics given by the three master equations. We find the validity condition for the TCL and NZ master equations. Based on the exact non-Markovian master equation, we analyze the regime of validity for the secular approximation in the time-convolutionless master equation and discuss the leading corrections of the nonsecular terms to the quantum dynamics, significant effects are found in the dynamics of the driven system.
\end{abstract}

PACS numbers: 03.65.Yz, 42.50.Lc

\section{INTRODUCTION}

The dynamics of open quantum systems [1-3] has attracted much attention and becomes active again in recent years due to its possible applications in quantum information science [4 9]. Indeed the study of coupled system-environment system is an long standing endeavor in many fields of physics including quantum optics 10 13], atomic optics [13 17] and condensed matter physics [3, 18, 19]. The coupling of the system to its environment leads to dissipation and dephasing with flows of energy or information from the system to the environment [2, 3$]$. The back flowing of information from the environment to the system determines the Markovianity of the dynamics.

Driven two-level model is available to effectively describe many actual physical systems, for example, a quantum bit in quantum information processing. Thus the theoretical analysis as well as the practical implementation of the driven two-level systems brings us a renewed topic. There are several ways to create a driven two-level system (or qubit) today by current quantum technologies, each exploits different approaches or in different quantum systems. For instance, by means of quantum optics and in microscopic quantum objects (electrons, ions, atoms) in traps, quantum dots, and quantum circuits 20 23]. Different implementations of qubit 24, 25] are subjected to different types of environmental noise [26], most environments are assumed Markovian [27, 28] and the dynamics of system was studied perturbatively in the literatures.

\footnotetext{
${ }^{*}$ Corresponding address: yixx@nenu.edu.cn
}

In recent years, an increasing interest has been paid to developing a non-Markovian generalization for open quantum system theory, some of them are formulated in terms of non-local time evolutions. There exist diverse formalisms for describing memory effects, including the generalization of the Lindblad master equation from time-independent dissipative rates to time-convoluted kernel functions. A wide class of both phenomenological and theoretical approaches were formulated for building and characterizing this type of master equations, which in turn lead to a completely positive map.

By means of the Feynman-Vernon influence functional theory [29 33], exact master equations describing the general non-Markovian dynamics of a wide range of open quantum system have been recently developed, e.g. quantum Brownian motion [32, 35, 36], single-mode cavity [37] and two entangled cavities [38, 39] with vacuum fluctuations, spin-boson model [40], coupled harmonic oscillators [41 43], quantum dot in nanostructures [44, 45], various nanodevices with time-dependent external control field 46], nanocavity systems including initial system-reservoir correlations [47], and photonic networks imbedded in photonic crystals [48, 49]. However, an exact master equation for driven systems are very rare.

Projection operator technique is other mean to study the open quantum system, both the time-convolutionless (TCL) [50 52] master equation and the NakajimaZwanzig (NZ) [53 55] master equation can be derived by this approach. The NZ approach provides us with a generalized master equation in which the time derivative of the density operator is connected to the past of the reduced density matrix through the convolution of the density operator and an appropriate integral kernel. While the TCL approach leads to a generalized master 
equation which is local in time. It seems that the NZ should run better than the TCL approach in describing the non-Markovian effect, since it takes into account the history of the reduced density matrix. However this is not the case as we will show later, examples in [2, 5663. confirm this point, namely, the exact dynamics of the open system can be described via a master equation with time-dependent decay rate, as in the well-known case of the Hu-Paz-Zhang generalized master equation [2, 32].

In the weak coupling limit, the non-Markovian master equation for a driven two-level system coupled to a bosonic reservoir at zero temperature has been derived and discussed in Ref. [62]. This derivation treat the system-environment coupling perturbatively, and hence it is available for weak system-environment couplings. In this paper, exploiting the Feynman-Vernon influence theory in the coherent state path integral formalism, we derive an exact non-Markovian master equation for the driven two-level system. The Feynman-Vernon influence theory enables us to treat the environment-system coupling non-perturbatively. The dynamics of the driven open two-level system, going beyond the TCL, NZ, and Markovian approximations, is governed by an effective action associated with the influence functional containing all the influences of the environment on the system. The exact master equation is available to examine the validity of those perturbative approaches applied to the TCL and NZ techniques. We show that the TCL approach works better than the NZ one, since the latter does not guarantee the positivity of the density matrix when the correlations in the reservoir become strong, while the former is available for a wider range of values of reservoir memory time.

The remainder of the paper is organized as follows. In Sec. II, we introduce a model to describe a driven two-level system subject to reservoir and give a detailed derivation of the influence functional for the model in the coherent state representation. In Sec. III, an exact non-Markovian master equation describing the evolution of the driven open two-level system is derived. In Sec. IV, a derivation of the second-order NZ master equation is presented and the characteristics of the second-order TCL derived in Ref. [2] are discussed, and then we give a comparison among the exact, TCL, and NZ master equations. In Sec. V, we investigate the validity of the secular approximation in Markovian and non-Markovian regimes, respectively. Discussions and conclusions are given in Sec. VI.

\section{ATOMIC COHERENT STATE PATH-INTEGRATE APPROACH TO THE DRIVEN OPEN TWO-LEVEL SYSTEM}

\section{A. Model Hamiltonian}

We start by considering a two-level system with Rabi frequency $\omega_{0}$ driven by an external laser of frequency $\omega_{L}$. The two-level atom is embedded in a bosonic reservoir at zero-temperature modeled by a set of infinite harmonic oscillators. In a rotating frame, the Hamiltonian of such a system (system plus environment) takes

$$
H=H_{S}+H_{E}+H_{I}
$$

with

$$
\begin{aligned}
& H_{S}=\Delta \cdot \sigma_{+} \sigma_{-}+\Omega \sigma_{x}, \\
& H_{E}=\sum_{k} \Omega_{k} a_{k}^{\dagger} a_{k}, \\
& H_{I}=\sum_{k} g_{k} \sigma_{+} a_{k}+\text { H.c. },
\end{aligned}
$$

where $\Delta=\omega_{0}-\omega_{L}, \Omega_{k}=\omega_{k}-\omega_{L}$, and $\sigma_{x}=\sigma_{+}+\sigma_{-}$. $\Omega$ is the driven strength, and H.c. stands for the Hermitian conjugation. $\sigma_{+}=|e\rangle\langle g|$ is the Pauli matrix. $a_{k}$ and $g_{k}$ are the annihilation operator and coupling constants, respectively. In the following we shall start with this Hamiltonian (1) and derive all master equations in this paper.

\section{B. Coherent state representation}

The starting point of analysis is to observe that the lowing and raising operators of the atomic transition operators $\sigma_{+}=|e\rangle\langle g|$ and $\sigma_{-}=|g\rangle\langle e|$ satisfy anticommutation rules similar to those of fermions, i.e.,

$$
\begin{aligned}
& \left\{\sigma_{-}, \sigma_{+}\right\}=|e\rangle\langle e|+| g\rangle\langle g| \equiv 1, \\
& \left\{\sigma_{-}, \sigma_{-}\right\}=\left\{\sigma_{+}, \sigma_{+}\right\}=0
\end{aligned}
$$

where $\{A, B\}=A B+B A$. Identifying the ground state $|g\rangle$ with the fermionic vacuum, we can therefore treat $\sigma_{+}$ and $\sigma_{-}$as fermionic creation and annihilation operators, respectively. Following Ref. [64], we introduce a couple of conjugate Grassmann variables $\zeta$ and $\bar{\zeta}$ imposing standard anticorrelation with the annihilation and creation operators of the system.

Therefore, coherent states are defined as a tensor product of states generated by exponentiated operation of a creation operator and a suitable label on a chosen fiducial state [29, 33, 65 67]

$$
|\mathbf{z}\rangle=\prod_{k}\left|z_{k}\right\rangle,\left|z_{k}\right\rangle=\exp \left(a_{k}^{\dagger} z_{k}\right)\left|0_{k}\right\rangle
$$

and

$$
|\zeta\rangle=\exp \left(\sigma_{+} \zeta\right)|g\rangle
$$

For bosonic coherent states defined in Eq. (41), the label $z_{k}$ is a complex number, and for atomic coherent states defined in Eq. (5), the label $\zeta$ is a Grassmannian or anticommuting number. A state of the combined atom-field 
system can be expanded in a direct product of coherent state

$$
|\mathbf{z} \zeta\rangle=|\mathbf{z}\rangle \otimes|\zeta\rangle .
$$

Atomic and bosonic coherent states possess the wellknown properties such as being nonorthogonal

$$
\begin{gathered}
\left\langle\mathbf{z} \mid \mathbf{z}^{\prime}\right\rangle=\exp \left(\sum_{k} \bar{z}_{k} z_{k}^{\prime}\right),\left\langle\zeta \mid \zeta^{\prime}\right\rangle=\exp \left(\bar{\zeta} \zeta^{\prime}\right), \\
a_{k}\left|z_{k}\right\rangle=z_{k}\left|z_{k}\right\rangle, \sigma_{-}|\zeta\rangle=\zeta|\zeta\rangle,
\end{gathered}
$$

where $\bar{z}_{k}$ and $\bar{\zeta}$ denote the conjugation of $z_{k}$ and $\zeta$, respectively. Despite their nonorthogonality, both types of coherent states form an over-complete basis set

$$
\int d \varphi(\mathbf{z})|\mathbf{z}\rangle\left\langle\mathbf{z}\left|=\int d \varphi(\zeta)\right| \zeta\right\rangle\langle\zeta|=1,
$$

where the integral measures are defined by $d \varphi(\mathbf{z})=$ $\prod_{k} \frac{\exp \left(-\bar{z}_{k} z_{k}\right) d^{2} z_{k}}{\pi}$, and $d \varphi(\zeta)=\exp (-\bar{\zeta} \zeta) d^{2} \zeta$. As shown, the bosonic coherent states we use here are not normalized, and the normalization factors are moved into the integration measures, which is similar to the Bargmann representation of the complex space. The application of the coherent state representation makes the evaluation of path integrals extremely simple. In the coherent state representation, the Hamiltonians of the system, the environment, and the interaction between them are expressed as, respectively

$$
\begin{aligned}
H_{S}(\bar{\zeta}, \zeta) & =\Delta \bar{\zeta} \zeta+\Omega(\bar{\zeta}+\zeta), \\
H_{E}(\overline{\mathbf{z}}, \mathbf{z}) & =\sum_{k} \Omega_{k} \bar{z}_{k} z_{k}, \\
H_{I}(\overline{\mathbf{z}}, \mathbf{z}, \bar{\zeta}, \zeta) & =\left(g_{k} \bar{\zeta} z_{k}+g_{k}^{*} \bar{z}_{k} \zeta\right) .
\end{aligned}
$$

With these notations, we will present a detailed derivation of the exact master equation for the reduced density matrix of the system in the following sections.

\section{The influence functional in coherent state representation}

Explicitly, the density matrix of the whole system (the system plus the environment) obeys the quantum Liouville equation, $i \partial \rho_{T}(t) / \partial t=\left[H, \rho_{T}(t)\right]$, which gives the formal solution

$$
\rho_{T}(t)=\exp (-i H t) \rho_{T}(0) \exp (i H t) .
$$

In the coherent state representation, by use of Eq. (9), $\rho_{T}(t)$ can be expressed as

$$
\begin{aligned}
& \left\langle\zeta_{f}, \mathbf{z}_{f}\left|\rho_{T}(t)\right| \zeta_{f}^{\prime}, \mathbf{z}_{f}\right\rangle \\
= & \int d \varphi\left(\mathbf{z}_{i}\right) d \varphi\left(\zeta_{i}\right) d \varphi\left(\mathbf{z}_{i}^{\prime}\right) d \varphi\left(\zeta_{i}^{\prime}\right)\left\langle\zeta_{f}, \mathbf{z}_{f} ; t \mid \zeta_{i}, \mathbf{z}_{i} ; 0\right\rangle \\
& \times\left\langle\zeta_{i}, \mathbf{z}_{i}\left|\rho_{T}(0)\right| \zeta_{i}^{\prime}, \mathbf{z}_{i}^{\prime}\right\rangle\left\langle\zeta_{i}^{\prime}, \mathbf{z}_{i}^{\prime} ; 0 \mid \zeta_{f}^{\prime}, \mathbf{z}_{f} ; t\right\rangle .
\end{aligned}
$$

Assume the initial density matrix be factorized into a direct product of the system and the environment state, i.e., $\rho_{T}(0)=\rho(0) \otimes \rho_{E}(0)[19]$, the reduced density matrix of the system is then given by

$$
\begin{aligned}
& \rho\left(\bar{\zeta}_{f}, \zeta_{f}^{\prime} ; t\right)=\int d \varphi\left(\mathbf{z}_{f}\right)\left\langle\zeta_{f}, \mathbf{z}_{f}\left|\rho_{T}(t)\right| \zeta_{f}^{\prime}, \mathbf{z}_{f}\right\rangle \\
& =\int d \varphi\left(\zeta_{i}\right) d \varphi\left(\zeta_{i}^{\prime}\right) \rho\left(\bar{\zeta}_{i}, \zeta_{i}^{\prime} ; 0\right) \cdot J\left(\bar{\zeta}_{f}, \zeta_{f}^{\prime} ; t \mid \bar{\zeta}_{i}, \zeta_{i}^{\prime} ; 0\right) .
\end{aligned}
$$

The next task is to determine the effective propagating function for the reduced density matrix [29, 30, 68],

$$
\begin{aligned}
J\left(\bar{\zeta}_{f}, \zeta_{f}^{\prime} ; t \mid \bar{\zeta}_{i}, \zeta_{i}^{\prime} ; 0\right)= & \int D^{2} \zeta D^{2} \zeta^{\prime} \exp \left\{i \left(S_{S}[\bar{\zeta}, \zeta]\right.\right. \\
& \left.\left.-S_{S}^{*}\left[\bar{\zeta}^{\prime}, \zeta^{\prime}\right]\right)\right\} F\left[\bar{\zeta}, \zeta, \bar{\zeta}^{\prime}, \zeta^{\prime}\right],
\end{aligned}
$$

with $S_{S}[\bar{\zeta}, \zeta]$ being the action of the system in the atomic coherent state representation, see Eq. (A.2). $F\left[\bar{\zeta}, \zeta, \bar{\zeta}^{\prime}, \zeta^{\prime}\right]$ is the influence functional which takes into account the back-action (in Eq. (A.1)) of the environment on the system.

Assume the environment be initially at zero temperature, i.e., the initial state of the environment takes,

$$
\rho_{E}=|0\rangle_{B B}\langle 0|,
$$

then the influence functional can be solved exactly and we have

$$
\begin{aligned}
F\left[\bar{\zeta}, \zeta, \bar{\zeta}^{\prime}, \zeta^{\prime}\right]= & \exp \left\{\int _ { t _ { 0 } } ^ { t } d \tau \int _ { t _ { 0 } } ^ { \tau } d \tau ^ { \prime } \left[f\left(\tau-\tau^{\prime}\right)\right.\right. \\
& \left(\bar{\zeta}^{\prime}(\tau)-\bar{\zeta}(\tau)\right) \zeta\left(\tau^{\prime}\right)+f^{*}\left(\tau-\tau^{\prime}\right) \\
& \left.\left.\bar{\zeta}^{\prime}\left(\tau^{\prime}\right)\left(\zeta(\tau)-\zeta^{\prime}(\tau)\right)\right]\right\},
\end{aligned}
$$

where

$$
\begin{aligned}
f\left(\tau-\tau^{\prime}\right) & =\sum_{k}\left|g_{k}\right|^{2} e^{-i \Omega_{k}\left(\tau-\tau^{\prime}\right)} \\
& =\int d \omega J(\omega) e^{-i\left(\omega-\omega_{L}\right)\left(\tau-\tau^{\prime}\right)}
\end{aligned}
$$

is called the dissipation-fluctuation kernel.

The details of derivation of Eq. (16) can be found in Appendix.

\section{THE EXACT NON-MARKOVIAN MASTER EQUATION}

We now derive the master equation for the reduced density matrix of the system. Since the effective action after tracing/integrating out the environmental degrees of freedom, (i.e., combining Eqs. (14) and (16) together) is in a quadratic form of the dynamical variables, the path integral (14) can be calculated exactly by making use of the stationary path method and Gaussian integrals 
[69, 70]. Substituting Eq. (A.2) into Eq. (14), we have

$$
\begin{aligned}
J\left(\bar{\zeta}_{f}, \zeta_{f}^{\prime} ; t \mid \zeta_{i}, \bar{\zeta}_{i}^{\prime} ; 0\right)= & \int D^{2} \zeta D^{2} \zeta^{\prime} \exp \left\{\frac { 1 } { 2 } \left[\bar{\zeta}_{f} \zeta(t)+\bar{\zeta}\left(t_{0}\right) \zeta_{i}\right.\right. \\
& \left.+\bar{\zeta}^{\prime}(t) \zeta_{f}^{\prime}+\bar{\zeta}_{i}^{\prime} \zeta^{\prime}\left(t_{0}\right)\right]-\int_{t_{0}}^{t} d \tau \frac{1}{2}[\bar{\zeta} \dot{\zeta} \\
& \left.-\overline{\bar{\zeta}} \zeta+\dot{\bar{\zeta}}^{\prime} \zeta^{\prime}-\bar{\zeta}^{\prime} \dot{\zeta}^{\prime}\right]+i H_{S}(\bar{\zeta}, \zeta) \\
& \left.-i H_{S}\left(\bar{\zeta}^{\prime}, \zeta^{\prime}\right)\right\} F\left[\bar{\zeta}, \zeta, \bar{\zeta}^{\prime}, \zeta^{\prime}\right]
\end{aligned}
$$

To calculate the path integral in Eq. (18), we use the stationary phase method [33, 34], which yields the equations of motion

$$
\begin{aligned}
& \dot{\zeta}(\tau)+i[\Omega+\Delta \cdot \zeta(\tau)]+\int_{t_{0}}^{\tau} d \tau^{\prime} f\left(\tau-\tau^{\prime}\right) \zeta\left(\tau^{\prime}\right)=0 \\
& \dot{\zeta}^{\prime}(\tau)+i\left[\Omega+\Delta \cdot \zeta^{\prime}(\tau)\right]-\int_{\tau}^{t} d \tau^{\prime} f\left(\tau-\tau^{\prime}\right) \zeta^{\prime}\left(\tau^{\prime}\right) \\
& +\int_{t_{0}}^{t} d \tau^{\prime} f\left(\tau-\tau^{\prime}\right) \zeta\left(\tau^{\prime}\right)=0
\end{aligned}
$$

subject to the boundary conditions $\zeta\left(t_{0}\right)=\zeta_{i}$ and $\zeta^{\prime}(t)=$ $\zeta_{f}^{\prime}$, respectively. $\quad \bar{\zeta}^{\prime}(\tau)$ and $\bar{\zeta}(\tau)$ denote the conjugates of $\zeta^{\prime}(\tau)$ and $\zeta(\tau)$, respectively. The equations for these conjugations can be obtained by first exchanging $\zeta(\tau)$ and $\zeta^{\prime}(\tau)$ in Eq. (19) and taking then a complex conjugate to these equations. The corresponding boundary conditions are $\bar{\zeta}^{\prime}\left(t_{0}\right) \equiv \bar{\zeta}_{i}^{\prime}$ and $\bar{\zeta}(t) \equiv \bar{\zeta}_{f}$. With these boundary conditions, we can get the solution of $\zeta(\tau)$ and $\zeta^{\prime}(\tau)$. For clarity, we illustrate these notations in Fig. 1, Noticing $t_{0} \leq \tau \leq t$, we keep in mind that $\zeta(t)$ in Fig. 1 (a) can be obtained by setting $\tau=t$ and $\zeta^{\prime}\left(t_{0}\right)$ in Fig. 1 (b) can be obtained by $\tau=t_{0}$. Fig. 1 (c) and (d) is similar, namely, $\bar{\zeta}^{\prime}(t)$ and $\bar{\zeta}^{\prime}\left(t_{0}\right)$ can be obtained with $\tau=t$ and $\tau=t_{0}$, respectively.

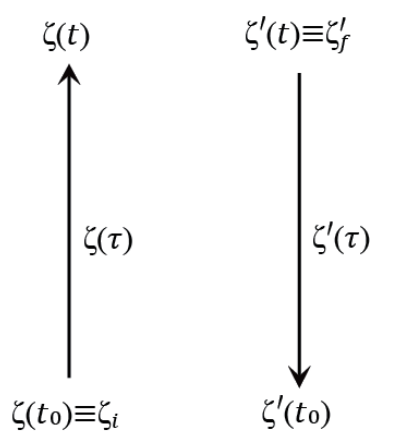

(a)

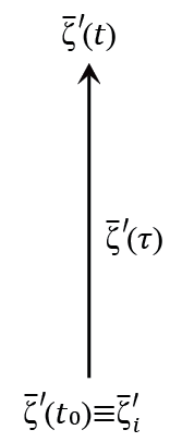

(c)

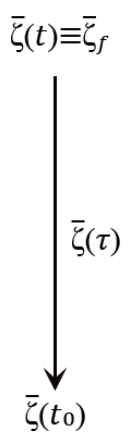

(d)
FIG. 1: (Color online) Schematic illustration of the four independent paths denoted by $\zeta(\tau), \zeta^{\prime}(\tau), \bar{\zeta}^{\prime}(\tau)$ and $\bar{\zeta}(\tau)$, respectively.

The solution of the integro-differential Eq. (19) can be expressed in terms of two complex functions $u(\tau)$ and $u_{1}(\tau)$ as

$$
\begin{aligned}
\zeta^{\prime}(\tau) & =u_{1}(\tau)\left[\zeta_{f}^{\prime}-\zeta(t)\right]+\zeta(\tau), \\
\zeta(\tau) & =u(\tau) \zeta_{i}+h(\tau),
\end{aligned}
$$

a similar transformation can be written down for their conjugate variables with the exchange of $\zeta$ with $\zeta^{\prime}$ for the boundary values $\bar{\zeta}(t)=\bar{\zeta}_{f}$ and $\bar{\zeta}^{\prime}\left(t_{0}\right)=\bar{\zeta}_{i}^{\prime}$. Substituting Eq. (20) into Eq. (19), we can obtain the equations of motion for $u(\tau), u_{1}(\tau)$ and $h(\tau)$

$$
\begin{gathered}
\dot{u}(\tau)+i \Delta \cdot u(\tau)+\int_{t_{0}}^{\tau} d \tau^{\prime} f\left(\tau-\tau^{\prime}\right) u\left(\tau^{\prime}\right)=0, \\
\dot{u}_{1}(\tau)+i \Delta \cdot u_{1}(\tau)-\int_{\tau}^{t} d \tau^{\prime} f\left(\tau-\tau^{\prime}\right) u_{1}\left(\tau^{\prime}\right)=0 \\
\dot{h}(\tau)+i \Delta \cdot h(\tau)+\int_{t_{0}}^{\tau} d \tau^{\prime} f\left(\tau-\tau^{\prime}\right) h\left(\tau^{\prime}\right)=-i \Omega,
\end{gathered}
$$

subject to the boundary conditions $u_{1}(t)=1, u\left(t_{0}\right)=1$ and $h\left(t_{0}\right)=0$ with $t_{0} \leq \tau, \tau^{\prime} \leq t$. By means of Laplace transform to Eq. (21), we can easily find that

$$
u_{1}(\tau)=u^{*}(t-\tau), h(\tau)=-i \Omega \int_{t_{0}}^{\tau} d \tau^{\prime} u\left(\tau-\tau^{\prime}\right)
$$

Now, we set $\tau=t_{0}$ in the first equation and $\tau=t$ in the second equation of Eq. (20), $\zeta(t)$ and $\zeta^{\prime}\left(t_{0}\right)$ can be expressed in terms of the boundary conditions $\zeta_{i}$ and $\zeta_{f}^{\prime}$

$$
\begin{aligned}
\zeta(t) & =u(t) \zeta_{i}+h(t), \\
\zeta^{\prime}\left(t_{0}\right) & =u^{*}(t)\left[\zeta_{f}^{\prime}-h(t)\right]+n(t) \zeta_{i},
\end{aligned}
$$

where $n(t)=1-|u(t)|^{2}$. Similarly, $\bar{\zeta}\left(t_{0}\right)$ and $\bar{\zeta}^{\prime}(t)$ can be obtained by exchanging $\zeta$ and $\zeta^{\prime}$ in Eq. (23) and by taking a complex conjugate to these equations. Finally, substituting these results with Eq. (20) into Eq. (18), we obtain the form of the propagating function for the reduced density matrix

$$
\begin{aligned}
J\left(\bar{\zeta}_{f}, \zeta_{f}^{\prime} ; t \mid \zeta_{i}, \bar{\zeta}_{i}^{\prime} ; 0\right)= & \exp \left\{u(t)\left[\bar{\zeta}_{f}-h^{*}(t)\right] \zeta_{i}+u^{*}(t) \bar{\zeta}_{i}^{\prime}\right. \\
& \times\left[\zeta_{f}^{\prime}-h(t)\right]+n(t) \bar{\zeta}_{i}^{\prime} \zeta_{i}+h(t) \bar{\zeta}_{f} \\
& \left.+h^{*}(t) \zeta_{f}^{\prime}-|h(t)|^{2}\right\}
\end{aligned}
$$

Notice that the pre-exponential factor in Eq. (24) is one, this is due to the fact that Eq. (24) is the result of integrating out fluctuations around the stationary path. Now we can derive the master equation by computing the time derivative of Eq. (13). First, from Eq. (24), we can write down the following identities

$$
\zeta_{i} J=\frac{1}{u}\left(\frac{\delta J}{\bar{\zeta}_{f}}-h J\right), \bar{\zeta}_{i}^{\prime} J=\frac{1}{u^{*}}\left(\frac{\delta J}{\zeta_{f}^{\prime}}-h^{*} J\right),
$$

which will be used to remove $\zeta_{i}$ and $\bar{\zeta}_{i}^{\prime}$ from the time derivative of $J$. After taking time derivative of Eq. (13) 
and substituting Eqs. (24) and (25) into it, we obtain the evolution equation

$$
\begin{aligned}
\frac{\partial \rho\left(\bar{\zeta}_{f}, \zeta_{f}^{\prime}\right)}{\partial t}= & m \bar{\zeta}_{f} \mathrm{P}_{1}+m^{*} \zeta_{f}^{\prime} \mathrm{P}_{2}-\left(m+m^{*}\right) \mathrm{P}_{3} \\
& +m^{*} h^{*} \mathrm{P}_{1}+m h \mathrm{P}_{2}-\dot{h}^{*} \mathrm{P}_{1}-\dot{h} \mathrm{P}_{2} \\
& -m h \bar{\zeta}_{f} \rho-m^{*} h^{*} \rho \zeta_{f}^{\prime}+\dot{h} \bar{\zeta}_{f} \rho \\
& +\dot{h}^{*} \rho \zeta_{f}^{\prime}
\end{aligned}
$$

where, $m(t) \equiv \frac{\dot{u}(t)}{u(t)}, \mathrm{P}_{1} \equiv \frac{\partial \rho}{\partial \bar{\zeta}_{f}}, \mathrm{P}_{2} \equiv \frac{\partial \rho}{\partial \zeta_{f}^{\prime}}, \mathrm{P}_{3} \equiv \frac{\delta^{2} \rho}{\partial^{2} \bar{\zeta}_{f} \zeta_{f}^{\prime}}$. By introducing the following functional differential relations in the coherent state representation [29, 44]

$\bar{\zeta}_{f} \mathrm{P}_{1} \leftrightarrow \sigma_{+} \sigma_{-} \rho(t), \mathrm{P}_{2} \zeta_{f}^{\prime} \leftrightarrow \rho(t) \sigma_{+} \sigma_{-}, \mathrm{P}_{3} \leftrightarrow \sigma_{-} \rho(t) \sigma_{+}$,

we arrive at an exact non-Markovian master equation

$$
\frac{d \rho(t)}{d t}=-i[H(t), \rho(t)]+\gamma(t)\left[2 \sigma_{-} \rho(t) \sigma_{+}-\left\{\sigma_{+} \sigma_{-}, \rho(t)\right\}\right],
$$

with the effective Hamiltonian containing the classical driven field

$$
H(t)=s(t) \sigma_{+} \sigma_{-}+r(t) \sigma_{+}+r^{*}(t) \sigma_{-} .
$$

The renormalized frequency $s(t)$ and the renormalized driving field $r(t)$ are results of the back-action of the environment. The time dependent dissipative coefficient $\gamma(t)$ describes the dissipative non-Markovian dynamics due to the interaction between the system and environment. All these time-dependent coefficients can be given explicitly,

$$
\begin{aligned}
s(t) & =\frac{i}{2}[m(t)-c . c .], \\
\gamma(t) & =-\frac{1}{2}[m(t)+c . c .], \\
r(t) & =i[\dot{h}(t)-h(t) m(t)],
\end{aligned}
$$

where $u(t)$ and $h(t)$ are determined by the integrodifferential equations of Eq. (21). The non-Markovian effect is fully manifested in the integral kernels in Eq. (21), which include the non-local time-correlation function $f(t)$ of the environment. The non-Markovian memory effect is coded into the homogenous non-local time integrals with the integral kernel. In addition, our derivation of the master equation is fully non-perturbative, which goes beyond the TCL, NZ and Markovian approximations and includes all effects resulting from the environment-system couplings.

\section{COMPARISON BETWEEN THE EXACT AND APPROXIMATE MASTER EQUATIONS}

\section{A. The Nakajima-Zwanzig and time-convolutionless master equations}

To derive the second-order perturbative master equation, we first go to the interaction picture, in which the effective Hamiltonian $H_{I}(t)$ in Eq. (2) can be rewritten as

$$
H_{I}(t)=\sigma_{-}(t) a^{\dagger}(t)+H . c .,
$$

where $\sigma_{-}(t)=U^{\dagger}(t) \sigma_{-} U(t), U(t)=e^{-i H_{S} t}, a^{\dagger}(t)=$ $\sum_{k} g_{k} a_{k}^{\dagger} e^{i \Omega_{k} t}$. The density operator $\bar{\rho}_{T}(t)$ of the whole system including the system and environment satisfies the following Liouville equation

$$
\dot{\bar{\rho}}_{T}(t)=-i\left[H_{I}(t), \bar{\rho}_{T}(t)\right] .
$$

Integrating the left and right sides of Eq. (32), we have

$$
\bar{\rho}_{T}(t)=\bar{\rho}_{T}\left(t_{0}\right)-i \int_{t_{0}}^{t} d t^{\prime}\left[H_{I}\left(t^{\prime}\right), \bar{\rho}_{T}\left(t^{\prime}\right)\right] .
$$

Substituting Eq. (33) into Eq. (32), we obtain

$$
\begin{aligned}
\dot{\bar{\rho}}_{T}(t)= & -i\left[H_{I}(t), \bar{\rho}_{T}(0)\right] \\
& -\int_{t_{0}}^{t} d t^{\prime}\left[H_{I}(t),\left[H_{I}\left(t^{\prime}\right), \bar{\rho}_{T}\left(t^{\prime}\right)\right]\right] .
\end{aligned}
$$

Tracing over the degrees of freedom of the environment, we can obtain the dynamical equation for the system density matrix $\bar{\rho}(t)=\operatorname{Tr}_{B} \bar{\rho}_{T}(t)$

$$
\begin{aligned}
\dot{\bar{\rho}}(t)= & -i \operatorname{Tr}_{R}\left[H_{I}(t), \bar{\rho}_{T}\left(t_{0}\right)\right] \\
& -\operatorname{Tr}_{R} \int_{t_{0}}^{t} d t^{\prime}\left[H_{I}(t),\left[H_{I}\left(t^{\prime}\right), \bar{\rho}_{T}\left(t^{\prime}\right)\right]\right] .
\end{aligned}
$$

Let us apply the Born approximation and assume that the reservoir stays in the vacuum state (15) in the dynamics, then we have

$$
\dot{\bar{\rho}}(t)=-\operatorname{Tr}_{R} \int_{t_{0}}^{t} d t^{\prime}\left[H_{I}(t),\left[H_{I}\left(t^{\prime}\right), \bar{\rho}\left(t^{\prime}\right) \otimes \rho_{E}\right]\right] .
$$

Notice that

$$
\begin{aligned}
\left\langle a(t) a^{\dagger}\left(t_{1}\right)\right\rangle & =f\left(t-t_{1}\right), \\
\left\langle a^{\dagger}(t) a^{\dagger}\left(t_{1}\right)\right\rangle & =\left\langle a(t) a\left(t_{1}\right)\right\rangle=\left\langle a^{\dagger}(t) a\left(t_{1}\right)\right\rangle=0,
\end{aligned}
$$

where $\langle A\rangle=\operatorname{Tr}_{B}\left\langle A \rho_{E}\right\rangle=\langle 0|A| 0\rangle_{B}$, and substituting Eq. (31) into Eq. (36), we have

$$
\dot{\bar{\rho}}(t)=\int_{t_{0}}^{t} d t^{\prime} f\left(t-t^{\prime}\right)\left[\sigma_{-}\left(t^{\prime}\right) \bar{\rho}\left(t^{\prime}\right), \sigma_{+}(t)\right]+H . c . .
$$

By transforming Eq. (38) back into the Schrödinger picture, we obtain

$$
\begin{aligned}
\dot{\rho}_{N Z}= & -i\left[H_{S}, \rho_{N Z}(t)\right]+\int_{t_{0}}^{t} d t^{\prime}\left\{f ( t - t ^ { \prime } ) \left[U\left(t-t^{\prime}\right)\right.\right. \\
& \left.\left.\times \sigma_{-} \rho_{N Z}\left(t^{\prime}\right) U^{\dagger}\left(t-t^{\prime}\right), \sigma_{+}\right]+H . c .\right\} .
\end{aligned}
$$

The non-Markovian master equation (39) is in the standard form of the Nakajima-Zwanzig (NZ) equation $\dot{\rho}(t)=$ 
$\int_{0}^{t} d t^{\prime} f\left(t, t^{\prime}\right) \rho\left(t^{\prime}\right)$ [53, 54], where the NZ kernel $f\left(t, t^{\prime}\right)$ is of the time-translationally-invariant form $f\left(t-t^{\prime}\right)$.

Note that Eq. (36) is in a form of delayed integrodifferential equation and thus it is a time-nonlocal master equation. It is worth reminding that the other systematically perturbative non-Markovian master equation that is local in time can be derived from the time-convolutionless projection operator formalism [2, 56, 57]. Now, we go to the details. Under a similar assumption, i.e., the factorized initial system-reservoir density matrix, the secondorder time-convolutionless master equation in the interaction picture can be obtained [2, 56 63$]$

$$
\dot{\bar{\rho}}(t)=-\operatorname{Tr}_{R} \int_{t_{0}}^{t} d t^{\prime}\left[H_{I}(t),\left[H_{I}\left(t^{\prime}\right), \bar{\rho}(t) \otimes \rho_{E}\right]\right] .
$$

Substituting Eq. (31) into Eq. (40) and using Eq. (37), we transform Eq. (38) back into the Schrödinger picture and obtain,

$$
\begin{aligned}
\dot{\rho}_{T C L}= & -i\left[H_{S}, \rho_{T C L}(t)\right]+\int_{t_{0}}^{t} d t^{\prime}\left\{f\left(t-t^{\prime}\right)\right. \\
& \times\left[\sigma_{-}\left(t^{\prime}-t\right) \rho_{T C L}(t) \sigma_{+}-\sigma_{+}\right. \\
& \left.\left.\times \sigma_{-}\left(t^{\prime}-t\right) \rho_{T C L}(t)\right]+H . c .\right\} .
\end{aligned}
$$

We note here that obtaining the time-convolutionless non-Markovian master equation perturbatively up to second order in the coupling by the use of the timeconvolutionless projection operator technique is equivalent to obtaining it by replacing $\bar{\rho}\left(t^{\prime}\right)$ with $\bar{\rho}(t)$ in Eq. (36) $[2,56$ 63]. One may wonder if the second order timenonlocal master equation (39) is more accurate than the second-order time-convolutionless master equation (41). In the following, using the exact master equation, we show that the TCL approach (41) works better than the NZ one (39) for a wide range of parameters.

\section{B. Comparison to the Nakajima-Zwanzig and time-convolutionless master equations}

We now analyze the characteristics of the damped driven two-level systems, by comparing the exact dynamics with that from the NZ and TCL master equations. Our purpose is to shed light on the performances of two master equations and to point out their ranges of validity. As stressed in the introduction, without the exact master equation, it is difficult to examine the range of validity for these master equations.

We assume that the system couples to a reservoir with detuning and the reservoir has a Lorentzian spectral density $2,62,71,72$

$$
J(\omega)=\frac{\Gamma}{2 \pi} \frac{\lambda^{2}}{\left(\omega-\omega_{0}+\delta\right)^{2}+\lambda^{2}},
$$

where $\delta=\omega_{0}-\omega_{c}$ is the detuning of $\omega_{c}$ to $\omega_{0}$, and $\omega_{c}$ is the center frequency of the cavity. It is worth noting that the parameter $\lambda$ defines the spectral width of the reservoir and is connected to the reservoir correlation time $\tau_{R}=\lambda^{-1}$. The parameter $\Gamma$ can be shown to be related to the decay of the system in the Markovian limit with a flat spectrum. The relaxation time scale is $\tau_{L}=\Gamma^{-1}$.

The Markovian dynamics usually describes a situation where the coupling strength between the system and the environment is very weak, and the characteristic correlation time $\tau_{R}$ of the environment is sufficiently shorter than that of the system $\tau_{L}$, i.e.,

$$
\tau_{R} \ll \tau_{L},
$$

equivalently, the spectrum of the reservoir takes $J(\omega)=$ $\frac{\Gamma}{2 \pi}$, which leads to a Markovian dynamics. The reservoir has no memory effect on the evolution of the system. Then according to Eq. (17), we have

$$
f(t)=\Gamma \delta(t) .
$$

Substituting Eq. (44) into the first equation of Eq. (21), we reduce the solution of $u(t)$ to

$$
u(t)=e^{-i \Delta t-\frac{\Gamma}{2} t},
$$

i.e., all the coefficients in Eq. ( 30 ) are constants,

$$
s(t)=\Delta, r(t)=\Omega, \gamma(t)=\Gamma .
$$

The exact master equation (28) is then reduced to Markovian master equation [2, 10, 73]

$$
\begin{aligned}
\frac{d \rho(t)}{d t}= & -i\left[\Delta \sigma_{+} \sigma_{-}+\Omega \sigma_{x}, \rho(t)\right]+\frac{\Gamma}{2}\left[2 \sigma_{-} \rho(t) \sigma_{+}\right. \\
& \left.-\left\{\sigma_{+} \sigma_{-}, \rho(t)\right\}\right],
\end{aligned}
$$

where the decoherence rates are time independent. This gives the standard Lindblad form for the Markovian dynamics. When

$$
\tau_{R} \geq \tau_{L}
$$

is satisfied, the strong non-Markovian effect plays an important role and the dynamics must be described by the exact master equation (28).

Now we calculate the two-time correlation functions $f\left(t-t^{\prime}\right)$ by substituting Eq. (42) into Eq. (17)

$$
f\left(t-t^{\prime}\right)=\frac{1}{2} \lambda \Gamma \exp \left[-(\lambda+i \Delta-i \delta)\left(t-t^{\prime}\right)\right] .
$$

It is clear that the bandwidth $\lambda$ is inversely proportional to the memory time of reservoir. For this correlation function $f\left(t-t^{\prime}\right)$, Eq. (21) can be easily solved by use of Eq. (49), the solution reads,

$$
u(t)=k(t) \times\left[\cosh \left(\frac{d t}{2}\right)+\frac{\lambda-i \delta}{d} \sinh \left(\frac{d t}{2}\right)\right],
$$

where $k(t)=e^{-(\lambda+2 i \Delta-i \delta) t / 2}$ and $d=\sqrt{(\lambda-i \delta)^{2}-2 \Gamma \lambda}$. 
In order to calculate $U(t)$ and $\sigma_{-}(t)$ in Eqs. (39) and (41), we calculate the eigenstates of the free system Hamiltonian $H_{S}$,

$$
\begin{aligned}
& \left|\phi_{\lambda 1}\right\rangle=\frac{1}{\sqrt{2}}(\sqrt{1+\sin \theta}|e\rangle+\sqrt{1-\sin \theta}|g\rangle), \\
& \left|\phi_{\lambda 2}\right\rangle=\frac{1}{\sqrt{2}}(\sqrt{1-\sin \theta}|e\rangle-\sqrt{1+\sin \theta}|g\rangle),
\end{aligned}
$$

the corresponding eigenvalues are $\lambda_{1}=\left(\Delta+W_{0}\right) / 2$ and $\lambda_{2}=\left(\Delta-W_{0}\right) / 2$. Here $W_{0}=\sqrt{\Delta^{2}+4 \Omega^{2}}, \theta=$ $\operatorname{acr} \tan (\Delta / 2 \Omega)$. Straightforward algebra yields,

$$
\begin{aligned}
\sigma_{-}(t) & =e^{i H_{S} t} \sigma_{-} e^{-i H_{S} t}=\sum_{j, k=1}^{2} \sigma_{j k} e^{i t\left(\lambda_{j}-\lambda_{k}\right)}\left|\phi_{\lambda j}\right\rangle\left\langle\phi_{\lambda k}\right|, \\
U(t) & =\sum_{j=1}^{2} e^{i \lambda_{j} t}\left|\phi_{\lambda j}\right\rangle\left\langle\phi_{\lambda j}\right|
\end{aligned}
$$

where $\sigma_{j k}=\left\langle\phi_{\lambda j}\left|\sigma_{-}\right| \phi_{\lambda k}\right\rangle$. Now let us concentrate on the average $\left\langle\sigma_{z}\right\rangle$, i.e., on the probability difference of finding the system in the atomic excited and ground levels. To examine the validity of the two approximate approaches we explore three different regimes by changing the width $\lambda$ of the Lorentzian spectral density. This investigation will allow us to estimate in which cases the non-Markovian master equations are efficient in the description of the system dynamics. Fig. 2 shows a comparison among the exact, TCL, and NZ master equations with large bandwidth $\lambda=25 \Gamma$. We find that the results given by the TCL (41) and NZ (39) are in good agreement with those obtained by the exact master equation (28) for any time scales. In this case, both TCL and NZ give a very good description for the dynamics. They indeed provide us with the same results, which are very close to the Markovian dynamics; see the discussion in Eq. (47). In addition, in such cases the TCL master equation which is easier to solve might be preferred to use because it is a time-local first order differential equations.

We set the same quantity $\lambda=\Gamma$ in Fig. 3. Clearly, the results given by the TCL (41) and NZ (39) are in good agreement with those obtained by the exact expression (28) in a short-time scale, but they deviate from each other in a long-time scale. Especially considering the long-time behavior, the NZ equation leads to a nonphysical result. For times longer than some critical values, the solution for the population difference $\left\langle\sigma_{z}\right\rangle$ cannot represent a physical result, because the absolute value of $\left\langle\sigma_{z}\right\rangle$ is larger than 1. We therefore can conclude that for this range of parameters the TCL equation gives a better description of the dynamics because it reflects all the qualitative characteristics of the exact expression.

One may wonder if this observation depends on the quantity plotted. To clarify this point, we plot the fidelity of the density matrix from the exact master equation to these from TCL and NZ master equations in Fig. 4, The results suggest that the TCL master equation is indeed better than the NZ for a wide range of parameters.

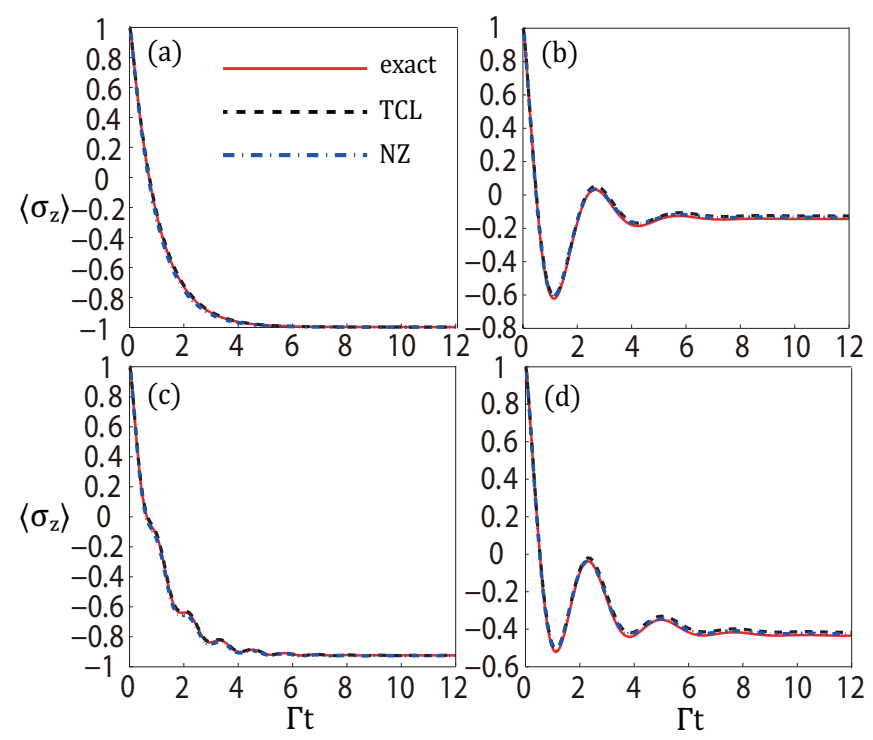

FIG. 2: (Color online) The time evolution of the population difference $\left\langle\sigma_{z}\right\rangle$ for the system initially in the excited state $|e\rangle$ versus the dimensionless parameter $\Gamma t$. The red line, blackdashed line, and blue-dashed-dotted line denote the exact Eq. (28), TCL Eq. (41), and NZ Eq. (39) master quations, respectively. The width of the Lorentzian spectral density is $\lambda=25 \Gamma$. The other parameters chosen are $\Delta=0.3 \Gamma, \Omega=$ $0.02 \Gamma, \delta=0.01 \Gamma$ for (a), $\Delta=0.3 \Gamma, \Omega=\Gamma, \delta=0.01 \Gamma$ for (b), $\Delta=5 \Gamma, \Omega=\Gamma, \delta=0.01 \Gamma$ for (c), $\Delta=\Gamma, \Omega=\Gamma, \delta=10 \Gamma$ for (d).

In Fig. [5 we choose the parameter $\lambda=0.05 \Gamma$, which, according to Eq. (49), corresponds to very strong reservoir correlations and very long memory effect. We find again that a good agreement among all the three approaches in the short-time scale, but in this case the TCL approximation works not so good. The dynamics of the TCL master equation (black-dashed line) does not succeed to follow the oscillations given by the exact expression (red line). The NZ approach has the same problem that it can not conserve the positivity of the density matrix (i.e., the absolute value of $\left\langle\sigma_{z}\right\rangle$ exceeds 1 ). Thus in this case two approximate methods are not suitable to describe the dynamics of the driven two-level system.

Before closing this section, we present a discussion on the function $f\left(\tau-\tau^{\prime}\right)$ in Eq. (17). Concretely, we examine mathematically the validity to extend the lower limit of the integration from 0 to $-\infty$. We will explore three different regimes characterized by the width $\lambda$ in the spectral density in the following.

In Fig. 6, we show a comparison between results with two different lower limits in the integration (17) with the spectral density given in Eq. (42), the simulation is performed for the exact dynamics described by Eq. (28). Fig. 6 (a) is for the integration with lower limit $-\infty$, which is slightly 


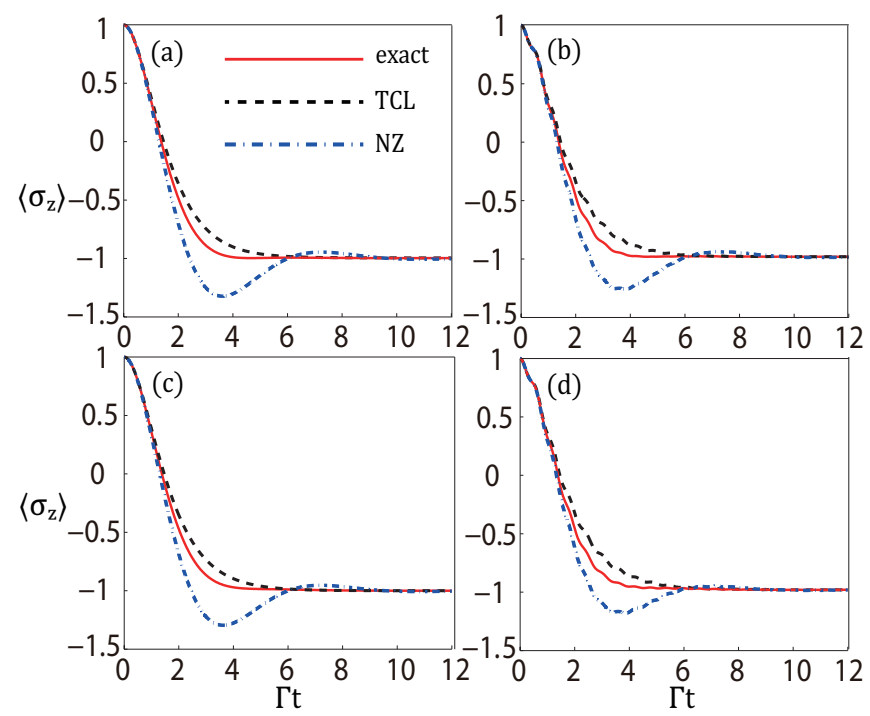

FIG. 3: (Color online) $\left\langle\sigma_{z}\right\rangle$ versus the dimensionless parameter $\Gamma t$. The width of the Lorentzian spectrum is $\lambda=\Gamma$. The results are obtained by the exact (red line), TCL (blackdashed line), and NZ (blue-dashed-dotted line) solutions. The other parameters chosen are $\Delta=0.3 \Gamma, \Omega=0.02 \Gamma, \delta=0.01 \Gamma$ for (a), $\Delta=10 \Gamma, \Omega=\Gamma, \delta=0.01 \Gamma$ for (b), $\Delta=10 \Gamma, \Omega=$ $0.02 \Gamma, \delta=0.2 \Gamma$ for (c), $\Delta=10 \Gamma, \Omega=\Gamma, \delta=0.2 \Gamma$ for $(\mathrm{d})$.

different from that with lower limit 0. In Fig. 6 (b) and (c), the results with lower limit $-\infty$ are in good agreement with that obtained with lower limit 0.

This numerical result can be explained as follows. When we change $\omega \rightarrow \omega-\omega_{L}$, Eq. (17) becomes

$$
f\left(\tau-\tau^{\prime}\right)=\int_{-\omega_{L}}^{\infty} d \omega J(\omega) e^{-i \omega\left(\tau-\tau^{\prime}\right)}
$$

with

$$
J(\omega)=\frac{\Gamma}{2 \pi} \frac{\lambda^{2}}{(\omega-\Delta+\delta)^{2}+\lambda^{2}},
$$

this tells us that the frequency $\omega_{L}$ affects only the lower limit of the integral (53) when $\Delta$ is fixed. Define $x_{ \pm}=\Delta-\delta \pm \lambda$ representing the position of half-height of the Lorentzian spectral density (54), we thought that the integral of $J(\omega)$ over $\omega$ from $-\infty$ to $\infty$ can be approximately replaced by the same integral but from $x_{-}$to $x_{+}$. With this approximation, we find that $x_{-}=-24.71 \Gamma$ and $\lambda=25 \Gamma$ in Fig. 6 (a). Clearly, $x_{-}$is much smaller than $-\omega_{L}$, thus the integral of $J(\omega)$ over $\omega$ from $x_{-}$ to $-\omega_{L}$ can not be ignored [see Fig. 6 (d)]. This explains the difference of the two curves in Fig. 6 (a). On the contrary, $\lambda=\Gamma, x_{-}=-0.71 \Gamma$ in Fig. 6 (b), and $\lambda=0.05 \Gamma, x_{-}=0.24 \Gamma$ in Fig. 6] (c). $x_{-}$
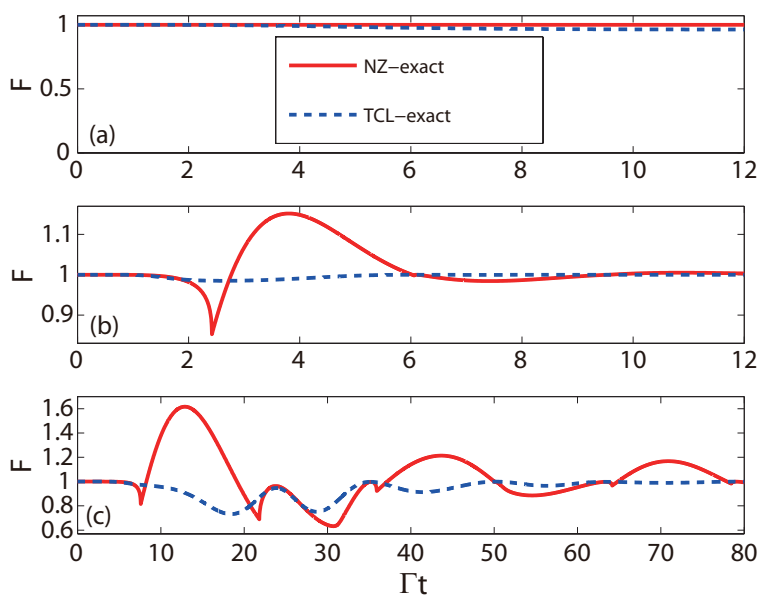

FIG. 4: (Color online) Comparison of the density matrices obtained by solving the TCL and NZ master equation with the one by exact master equation. We quantify the difference by the fidelity defined by $F\left(\rho_{1}, \rho_{2}\right)=\operatorname{Tr} \sqrt{\rho_{1}^{\frac{1}{2}} \rho_{2} \rho_{1}^{\frac{1}{2}}}$. The results show that the density matrix given by TCL is always better than that given by NZ master equation. The parameters in (a),(b) and (c) are chosen as the same as in Fig 2f(a), Fig 3 f(a) and Fig 5 . (a), respectively.

is larger than $-\omega_{L}$ in both cases of (b) and (c). Thus, the integral from $-\omega_{L}$ to $x_{-}$can be ignored [see Fig. 6 (e) and (f)]. As a result, the two lines in both (b) and (c) are in good agreement.

The above discuss suggests that it is reasonable to extend the lower limit of the integral of Lorentzian spectral $J(\omega)$ from 0 to $-\infty$.

\section{VALIDITY OF SECULAR APPROXIMATION IN TIME-CONVOLUTIONLESS MASTER EQUATIONS}

Taking advantage of the exact expression for the dissipative dynamics of the open driven two-level system, we have shown that the TCL approach can reveal all the characteristics of the non-Markovian dynamics for a range of parameters much wider than the results that the $\mathrm{NZ}$ equation gives, this is physically reasonable, since the latter may violate the positivity condition on the density matrix for the reservoir correlations which are not very strong. Therefore through comparing with the exact non-Markovian master equation (28), we can investigate the validity of the secular approximation based on time-convolutionless master equation (41).

We now use the orthonormalized basis (51) and these relations (52) to derive explicitly the time-convolutionless master equations (41) as follows

$$
\dot{\rho}=-i\left[H_{S}-H_{1}, \rho\right]+D(\rho)+D_{1}(\rho),
$$




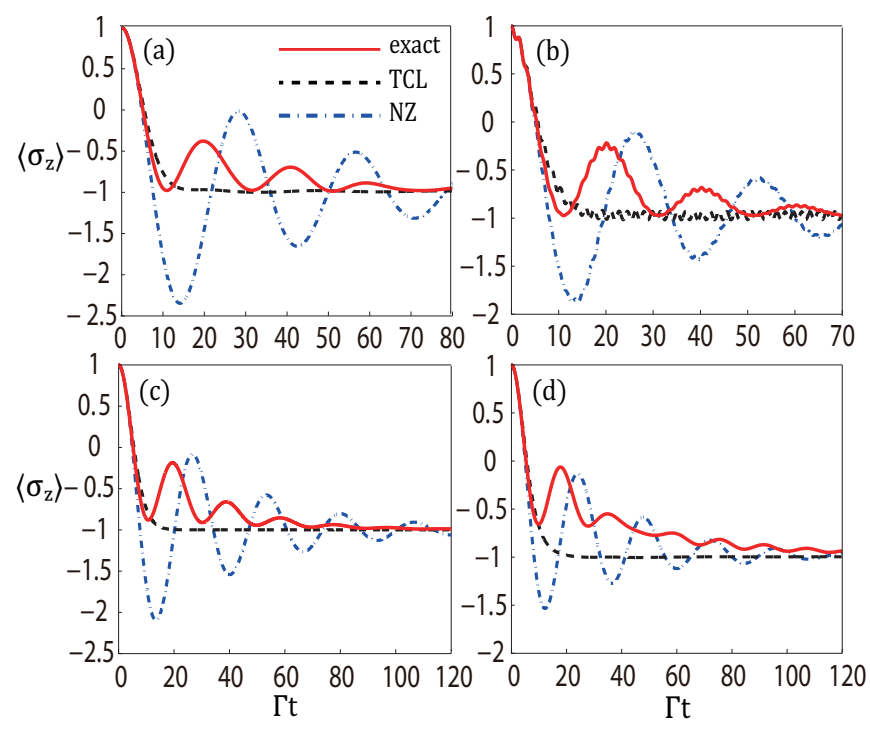

FIG. 5: (Color online) $\left\langle\sigma_{z}\right\rangle$ versus time $\Gamma t$. The results are obtained by the exact (red line), TCL (black-dashed line), and NZ (blue-dashed-dotted line) solutions. The parameters chosen are $\lambda=0.05 \Gamma, \Delta=0.3 \Gamma, \Omega=0.02 \Gamma, \delta=0.01 \Gamma$ for (a), $\Delta=3.5 \Gamma, \Omega=0.4 \Gamma, \delta=0.01 \Gamma$ for (b), $\Delta=10 \Gamma, \Omega=$ $0.02 \Gamma, \delta=0.08 \Gamma$ for $(\mathrm{c}), \Delta=0.3 \Gamma, \Omega=0.02 \Gamma, \delta=0.14 \Gamma$ for (d).

with

$$
H_{1}=g_{0}^{2} Q_{0}(t) S_{z}^{2}+g_{2}^{2} Q_{+1}(t) S_{-} S_{+}+g_{1}^{2} Q_{-1}(t) S_{+} S_{-},
$$

which describes a small shift in the energy of the twolevel system. The above new operators are defined as $S_{-}=\left|\phi_{\lambda 2}\right\rangle\left\langle\phi_{\lambda 1}\left|, S_{+}=\right| \phi_{\lambda 1}\right\rangle\left\langle\phi_{\lambda 2}\right|$, and $S_{z}=\left|\phi_{\lambda 1}\right\rangle\left\langle\phi_{\lambda 1}\right|-$ $\left|\phi_{\lambda 2}\right\rangle\left\langle\phi_{\lambda 2}\right|$, then the dissipative superoperator $D(\rho)$ in Eq. (55) can be written in a Lindblad form

$$
\begin{aligned}
D(\rho)= & g_{1}^{2} P_{-1}(t)\left[2 S_{-} \rho S_{+}-\left\{S_{+} S_{-}, \rho\right\}\right] \\
& +g_{2}^{2} P_{+1}(t)\left[2 S_{+} \rho S_{-}-\left\{S_{-} S_{+}, \rho\right\}\right] \\
& +g_{0}^{2} P_{0}(t)\left[2 S_{z} \rho S_{z}-\left\{S_{z}^{2}, \rho\right\}\right]
\end{aligned}
$$

where the coefficients $g_{0}=\Omega / W_{0}, g_{1}=\left(W_{0}+\Delta\right) /\left(2 W_{0}\right)$, $g_{2}=\left(W_{0}-\Delta\right) /\left(2 W_{0}\right), W_{0}=\sqrt{\Delta^{2}+4 \Omega^{2}}$. The second dissipator $D_{1}(\rho)$ in Eq. (55) has a more complicated form and contains the contribution of the so-called nonsecular terms,

$$
\begin{aligned}
D_{1}(\rho)= & g_{0} R_{0}(t)\left[g_{2}\left(S_{z} \rho S_{-}-S_{-} S_{z} \rho\right)+g_{1}\left(S_{+}\right.\right. \\
& \left.\left.\times S_{z} \rho-S_{z} \rho S_{+}\right)\right]+g_{2} R_{1}(t)\left[g _ { 0 } \left(S_{+} \rho S_{z}\right.\right. \\
& \left.\left.-S_{z} S_{+} \rho\right)-g_{1} S_{+} \rho S_{+}\right]+g_{1} R_{-1}(t)\left[g_{0}\right. \\
& \left.\times\left(S_{z} S_{-} \rho-S_{-} \rho S_{z}\right)-g_{2} S_{-} \rho S_{-}\right]+H . c . .
\end{aligned}
$$

For TCL master equations, the non-Markovian effects are contained in the time-dependent coefficients $P_{m}(t), Q_{m}(t)$, and $R_{m}(t)$, with $m \in\{+, 0,-\}$. The time-
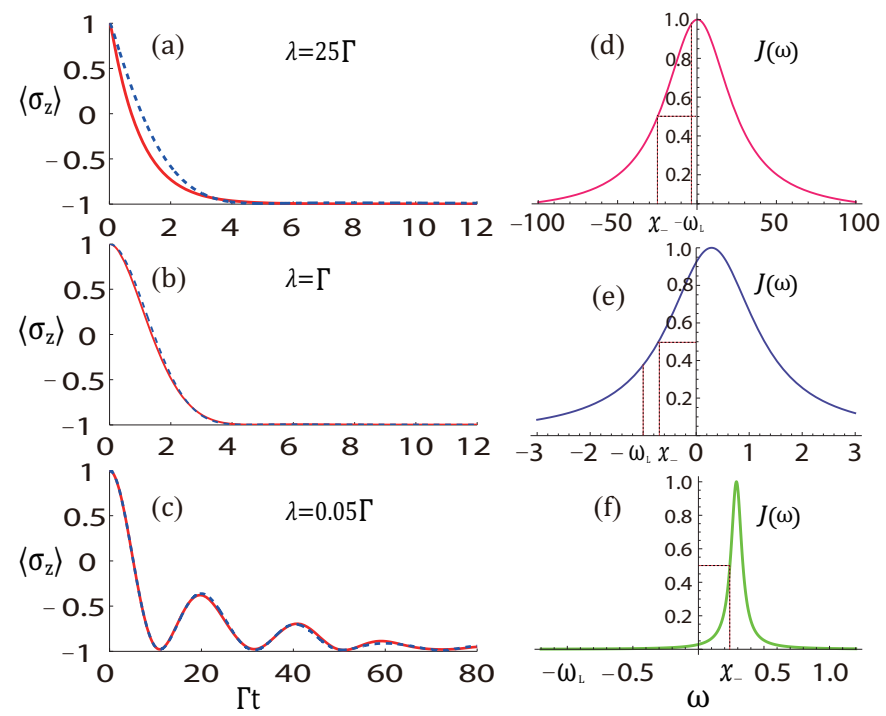

FIG. 6: (Color online) $\left\langle\sigma_{z}\right\rangle$ given by the exact master equation (28) as a function of time. The purpose of this figure is to show the difference in $\left\langle\sigma_{z}\right\rangle$ caused by different lower limits of the integral of kernel (53). The red and blue-dashed lines correspond to lower limits $-\omega_{L}$ and $-\infty$, respectively. The Lorentzian spectral density $J(\omega)$ (in units of $\Gamma / 2 \pi$ ) in (d), (e) and (f) correspond respectively to results shown in (a), (b) and (c). $x_{-}$denotes the left location of the half-height of the spectral density. The parameters in (a), (b) and (c) are chosen as the same as in Fig,2. (a), Fig.3-(a) and Fig.5-(a), respectively. Notice that $\Delta=\omega_{0}-\omega_{L}=0.3 \Gamma$ in Eq. (2), we set $\omega_{0}=1.3 \Gamma$ and $\omega_{L}=\Gamma$.

dependent coefficient reads

$$
R_{m}(t)=\int_{0}^{t} d t^{\prime} \int d \omega J(\omega) \exp \left[i\left(M_{m}-\omega\right)\left(t-t^{\prime}\right)\right]
$$

where $M_{m}=\omega_{L}-m W_{0}$. The other coefficients take $P_{m}(t)=\operatorname{Re}\left[R_{m}(t)\right]$ and $Q_{m}(t)=-\operatorname{Im}\left[R_{m}(t)\right]$. Conventionally, the nonsecular terms included in the dissipator $D_{1}(\rho)$ are neglected in the secular approximation. In order to investigate the effects of the nonsecular terms on the non-Markovian dynamics, we focus on two regimes identified by the mutual relationship between the system characteristic time and the reservoir correlation time.

The time-dependent coefficient (59) for the driven twolevel system in a Lorentzian reservoir can be calculated explicitly using Eq. (49)

$$
R_{m}(t)=\frac{\Gamma \lambda}{\lambda+i N_{m}}\left\{1-\exp \left[-\left(\lambda+i N_{m}\right) t\right]\right\}
$$

with

$$
N_{m}=\Delta-\delta+m W_{0}
$$


We can see from Eq. (60) that when $\min \left[\left|N_{+}\right|,\left|N_{0}\right|,\left|N_{-}\right|\right] \gg \lambda$, namely, the relaxation time $\tau_{R}=\lambda^{-1}$ of the reservoir correlation is very large compared to the typical timescale defined as $\tau_{S}=\left[\min \left(\left|N_{+}\right|,\left|N_{0}\right|,\left|N_{-}\right|\right)\right]^{-1}$, i.e.

$$
\tau_{R} \gg \tau_{S}
$$

is satisfied, oscillating terms (58) (that containing $R_{m}(t)$ ) may be neglected as $t$ increases, since rapid oscillations have no contribution to the dynamics on the timescale of the relaxation, this constitutes the secular approximation.

When

$$
\tau_{R} \leq \tau_{S},
$$

we cannot neglect the nonsecular terms (58) in the master equation (55) in the dynamics of the driven two-level system. Therefore in this case, we can no longer obtain a simple expression for the system. The master equation of the system is no longer in the time-dependent Lindblad form.

Examining Eqs. (43) and (62), we can summarize the comparison of the nonsecular with the secular approximation in the following Table —. which shows the validity regimes for secular and nonsecular approximation in TCL, Markovian and non-Markovian regimes, respectively. From Table I] we can divide the time dependent

TABLE I: Comparison of regimes of secular and nonsecular approximation in TCL for Markovian and non-Markovian

\begin{tabular}{|c|c|c|}
\hline & $\tau_{\mathrm{R}} \gg \tau_{\mathrm{S}}$ & $\tau_{\mathrm{R}} \leq \tau_{\mathrm{S}}$ \\
\hline $\begin{array}{r}\text { Markovian : } \\
\alpha \rightarrow \tau_{\mathrm{R}} \ll \tau_{\mathrm{L}}\end{array}$ & I secular regime & II nonsecular regime \\
\hline $\begin{array}{c}\text { non-Markovian } \\
\beta \rightarrow \tau_{\mathrm{R}} \geq \tau_{\mathrm{L}}\end{array}$ & III secular regime & IV nonsecular regime \\
\hline
\end{tabular}
regimes, respectively.

dynamics into two regimes, labeled by $\alpha$ and $\beta$, i.e., Markovian and non-Markovian regimes, respectively. In regime $\alpha$, i.e., Markovian regime, we can see that the results given by the regime I under the secular approximation in the TCL Eq. (55) are in good agreement with those obtained by the exact master equation Eq. (28) when the weak coupling condition (43) and the secular approximation (62) are simultaneously satisfied [see Figs. 7 (a), 7 (c), and 7 (e)]. When the parameters simultaneously satisfy Eqs. (43) and (663) [see Figs. 7 (b), 7(d), and 7 (f)], i.e., the regime (II), the dynamics of the TCL master equation (55) involving the nonsecular terms Eq. (58) are in good agreement with those obtained by the exact expression (28), but the results obtained by the secular approximation have serious deviations from those obtained by the exact solution Eq. (28). This difference

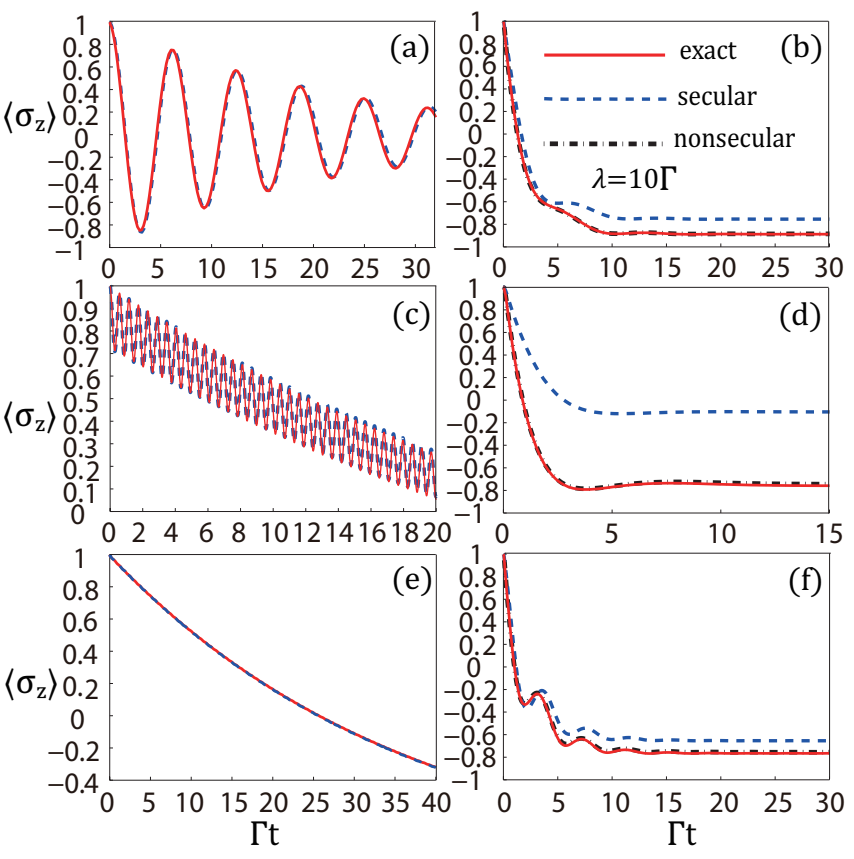

FIG. 7: (Color online) This plot shows the comparison of the secular approximation (regime I) $[(\mathrm{a}),(\mathrm{c})$, and (e)] and nonsecular terms (regime II) [(b), (d), and (f)] in Markovian regime $\alpha$ in Table \. The red line, blue-dashed line, and black dashed-dotted line denote the exact expression Eq. (28), the secular approximation Eq. (55) neglecting the nonsecular terms (58), and the nonsecular Eq. (55) containing (58), respectively. Parameters chosen are $\lambda=10 \Gamma, \Delta=0, \Omega=$ $0.5 \Gamma, \delta=40 \Gamma$ for (a), $\Delta=0.5 \Gamma, \Omega=0.2 \Gamma, \delta=10 \Gamma$ for (b), $\Delta=10 \Gamma, \Omega=2 \Gamma, \delta=60 \Gamma$ for $(\mathrm{c}), \Delta=0.1 \Gamma, \Omega=0.2 \Gamma, \delta=5 \Gamma$ for $(\mathrm{d}), \Delta=10 \Gamma, \Omega=0.2 \Gamma, \delta=60 \Gamma$ for $(\mathrm{e}), \Delta=\Gamma, \Omega=$ $0.5 \Gamma, \delta=10 \Gamma$ for $(\mathrm{f})$.

comes from the nonsecular terms (58), which are ignored in the regime (II).

Examining the non-Markovian regime labeled by $\beta$ in Table II we find that the results given by the secular approximation Eq. (57) in the regime III are in good agreement with those obtained by the exact expression Eq. (28) when the strong coupling condition (48) and the secular approximation (62) are simultaneously satisfied [see Figs. 8 (a), 8 (c), and 8 (e)]. When the parameters satisfy simultaneously Eqs. (48) and (63) [see Figs. 8(b), 8 (d), and 8 (f)], i.e., in the regime IV, the dynamics of the TCL master equation (55) involving the nonsecular terms Eq. (58) are in good agreement with those obtained by the exact one (28). However, the results obtained by the secular approximation have serious deviations from the exact solution Eq. (28). The same observation can be found in the regime II.

From Figs. 7 and 8 , we can learn that the nonMarkovian effect occurs when $\lambda$ is small. The nonMarkovian regime $\beta$ transits to the Markovian regime $\alpha$ when $\lambda$ is large. Therefore by manipulating $\lambda$ we can 


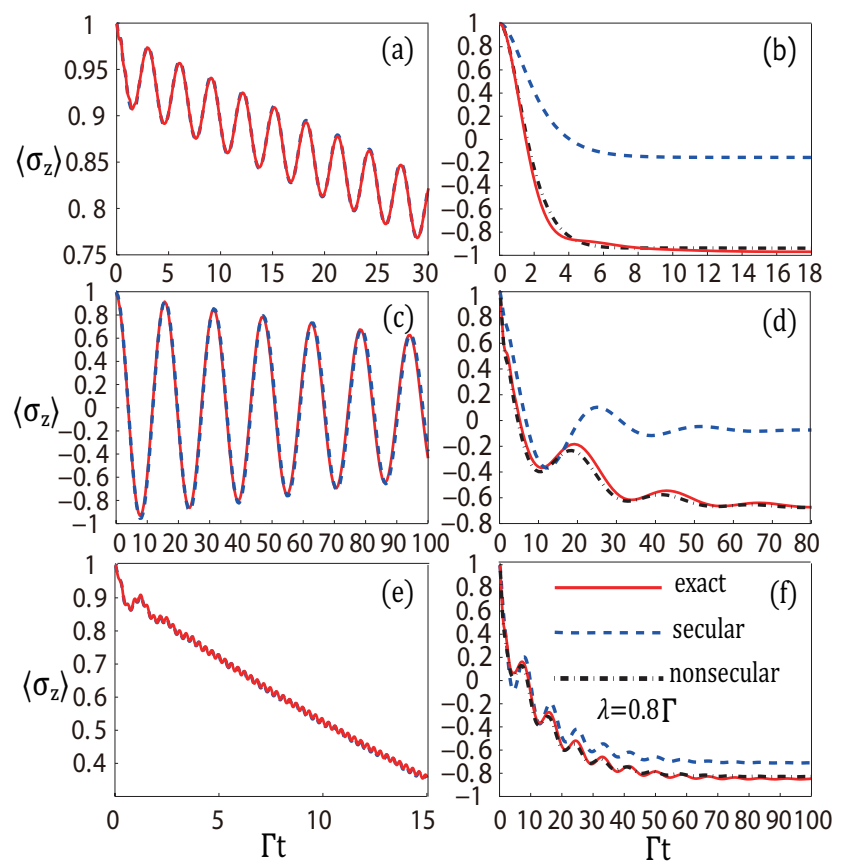

FIG. 8: (Color online) This plot shows the comparison of the secular approximation (regime III) [(a), (c), and (e)] with nonsecular terms (regime IV) [(b), (d), and (f)] in non-Markovian regime $\beta$ in Table \. The red line, blue-dashed line, and black dashed-dotted line denote the exact master equation Eq. (28), the secular approximation Eq. (55) neglecting the nonsecular terms (58), and the nonsecular Eq. (55) containing (58), respectively. Parameters chosen are $\lambda=0.8 \Gamma, \Delta=2 \Gamma, \Omega=$ $0.2 \Gamma, \delta=15 \Gamma$ for (a), $\Delta=0.04 \Gamma, \Omega=0.06 \Gamma, \delta=0.4 \Gamma$ for (b), $\Delta=0, \Omega=0.2 \Gamma, \delta=10 \Gamma$ for (c), $\Delta=0.05 \Gamma, \Omega=$ $0.1 \Gamma, \delta=1.8 \Gamma$ for $(\mathrm{d}), \Delta=20 \Gamma, \Omega=\Gamma, \delta=5 \Gamma$ for $(\mathrm{e})$, $\Delta=0.5 \Gamma, \Omega=0.2 \Gamma, \delta=2.5 \Gamma$ for (f).

control the crossover from non-Markovian to Markovian processes and vice versa. This provides us with a method to manipulate the non-Markovian dynamics in the driven two-level system.

Now we turn to discuss the positivity and complete positivity of the reduced dynamics given by the TCL master equation. The non-Markovian TCL master equation derived in this paper is not of the Lindblad form, even in the secular regime discussed in Sec.V, therefore, both the positivity and the complete positivity of the reduced dynamics can not be guaranteed. In other words, the Lindblad-Gorini-KossakowskiSudarshan theorem [74, 75] that ensures the positivity can not be satisfied in general, indicating that the dynamics given by the TCL master equation might not be physical for all range of parameters.

Nevertheless, the parameters chosen (in fact, it is wide range of parameters) in this paper assure the positivity of the reduced dynamics given by the TCL master equations. This can be understood as follows. For the driven qubit in the TCL approximation, the necessary and suf- ficient condition for complete positivity and positivity is given by (for details, see Ref. [62])

$$
2 \alpha(t)+\beta(t) \geq 0
$$

where

$$
\begin{aligned}
& \alpha(t)=2 \int_{0}^{t} d \tau\left[g_{1}^{2} P_{-1}(\tau)+g_{2}^{2} P_{+1}(\tau)+4 g_{0}^{2} P_{0}(\tau)\right], \\
& \beta(t)=4 \int_{0}^{t} d \tau\left[g_{1}^{2} P_{-1}(\tau)+g_{2}^{2} P_{+1}(\tau)\right] .
\end{aligned}
$$

Now back to the Sec. IV, we stress that the necessary and sufficient condition (64) for complete positivity is satisfied for the parameters chosen in Fig. (3) and (5) (not for a very long time). Therefore, for a wide range of parameters, the complete positivity of the reduced dynamics is guaranteed. Hence our conclusion, i.e., the TCL equation gives a better description of the dynamics, holds true for a wide range of parameters. It is important to remind that theoretical descriptions of nonMarkovian open quantum systems are often based on a series of assumptions and approximations without which it would not be possible to tackle the problem of the description of the dynamics in simple analytic terms. But those approximations plague almost all approximated reduced dynamics and lead them to break the complete positivity required for reduced dynamics. Therefore the observation here is available for short times and certain ranges of parameters.

\section{THE CASE WITH NON-LORENTZIAN SPECTRUM}

Note that the spectral density $J_{S B}(\omega)$ is proportional to the imaginary part of the dynamical susceptibility $\tilde{\chi}(\omega)$ of a damped harmonic oscillator, in this section, we present a numerical simulation for $\left\langle\sigma_{z}(t)\right\rangle$ adapting a different spectral density, e.g., spin-boson spectral density [3, 76],

$$
J_{S B}(\omega)=\frac{1}{M} \frac{\omega \lambda}{\left(\omega^{2}-\omega_{0}^{2}\right)^{2}+\omega^{2} \lambda^{2}} .
$$

In Fig. (9), we plot the time evolution of the population difference $\left\langle\sigma_{z}\right\rangle$ for three typical spectral width $\lambda$. Interestingly, in Fig. 9 (a), i.e., for large $\lambda=25 \Gamma$ the population difference $\left\langle\sigma_{z}\right\rangle$ decays monotonically for both spin-boson and Lorentzian spectral density, the difference is that the former decay more slowly than the latter. This corresponds to the Markovian case, see the discussion in Eq. (47). For $\lambda=\Gamma$, small oscillations can be observed in the case with spin-boson spectral density, while it is not obvious in the case with Lorentzian spectral density [see Fig. 9 (b)]. For small $\lambda=0.05 \Gamma$, oscillations in the population difference can be found in both cases with 
spin-boson spectral density and Lorentzian spectral density[see Fig. 9 (c)]. These oscillations correspond to a rapid exchange of energy and information between the two-level atom and reservoir.
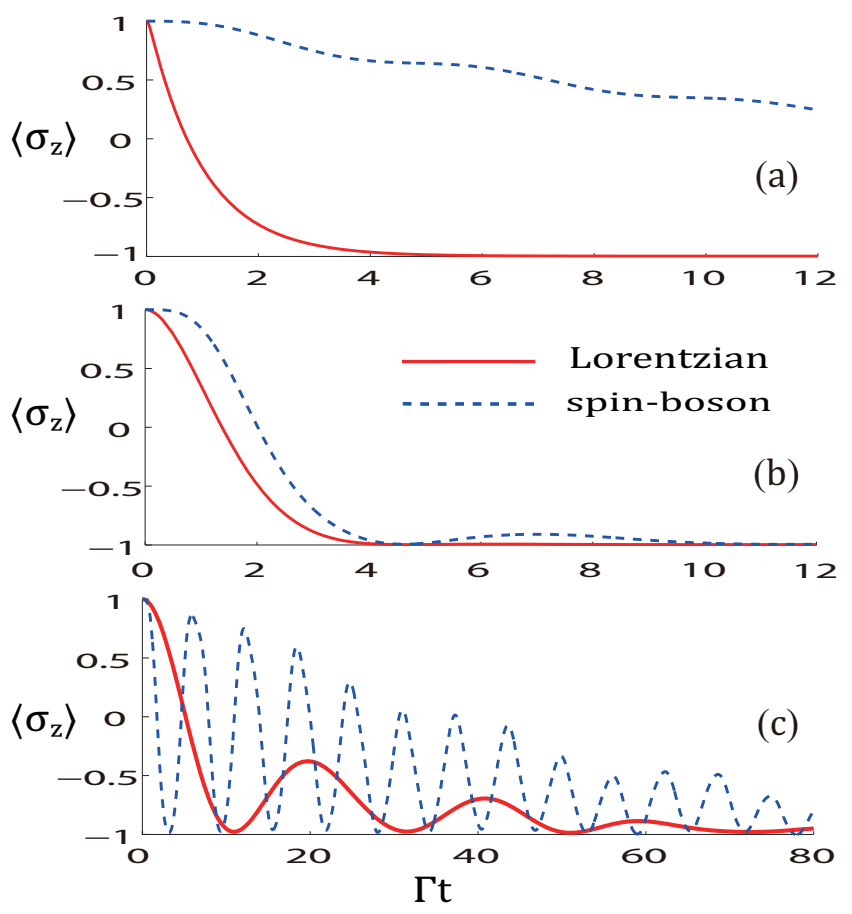

FIG. 9: (Color online) This plot shows the comparison of the exact dynamics (28) for Lorentzian (red-line) and spinboson(blue-dashed line) spectral density. The parameters in (a), (b) and (c) are chosen as the same as in Fig 2 (a), Fig 3 (a) and Fig 5 (a), respectively. The other parameter chosen is $M=5 \Gamma$.

Spectral density is a key feature for environments. It characterizes the correlation among the particles in the environment and determines the dynamics of open system, as we show in this section.

\section{CONCLUSION}

For a driven two-level quantum system, secular and weak coupling approximations break down when the system-environment coupling varies significantly on the scale of the Rabi frequency. In this paper, we avoid these approximations and have studied the non-Markovian dynamics of the driven two-level system coupled to a bosonic reservoir at zero temperature. Making use of the Feynman-Vernon influence functional theory in the coherent state representation, we derive an exact nonMarkovian master equation for the driven two-level system. We compare this exact master equation with the other equations describing non-Markovian dynamics, i.e., the Nakajima-Zwanzig and the time-convolutionless nonMarkovian master equation, it is found that the TCL approach is valid for a range of parameters much wider than the NZ master equation. This is reasonable since the latter may violate the positivity of dynamical map when the correlation in the reservoir is strong. By using the exact master equation, we also have given the analytical condition of validity of the secular approximation and show how it depends on the environmental spectral density, we found that the nonsecular terms have significant corrections to results obtained by the secular approximation when the relaxation time of the environment is less than or equal to that of the system, i.e. $\tau_{R} \leq \tau_{S}$.

The limitation of this representation is the state of the bath, here we only consider the bath initially at vacuum. Although the zero temperature case is problematic for getting reduced dynamics as the bath correlation functions may decay slowly, the zero-temperature reservoir is a good approximation for many problems in physics. For the reservoir initially at thermal states, the question becomes complicated, since the influence functional in the Feynman-Vernon influence functional theory is very involved.

\section{ACKNOWLEDGMENTS}

This work is supported by the NSF of China under Grants No. 11175032.

\section{Appendix: DERIVATION OF THE INFLUENCE FUNCTIONAL}

The propagating function controlling the time evolution of the reduced density matrix is given by Eq. (14), where the generalized Feynman-Vernon influence functional is defined by

$$
\begin{aligned}
F\left[\bar{\zeta}, \zeta, \bar{\zeta}^{\prime}, \zeta^{\prime}\right]= & \int d \varphi\left(\mathbf{z}_{f}\right) d \varphi\left(\mathbf{z}_{i}\right) d \varphi\left(\mathbf{z}_{i}^{\prime}\right) D^{2} \mathbf{z} D^{2} \mathbf{z}^{\prime} \\
& \times \rho_{E}\left(\overline{\mathbf{z}}_{i}, \mathbf{z}_{i}^{\prime} ; 0\right) \exp \left\{i \left(S_{E}[\overline{\mathbf{z}}, \mathbf{z}]\right.\right. \\
& -S_{E}^{*}\left[\overline{\mathbf{z}}^{\prime}, \mathbf{z}^{\prime}\right]+S_{I}[\overline{\mathbf{z}}, \mathbf{z}, \bar{\zeta}, \zeta] \\
& \left.\left.-S_{I}^{*}\left[\overline{\mathbf{z}}^{\prime}, \mathbf{z}^{\prime}, \bar{\zeta}^{\prime}, \zeta^{\prime}\right]\right)\right\}
\end{aligned}
$$


where $S_{S}, S_{I}$ and $S_{E}$ are the actions corresponding to $H_{S}, H_{I}$ and $H_{E}$, respectively,

$$
\begin{aligned}
S_{S}[\bar{\zeta}, \zeta]= & -i\left[\bar{\zeta}_{f} \zeta(t)+\bar{\zeta}\left(t_{0}\right) \zeta_{i}\right] / 2+\int_{t_{0}}^{t} d \tau\{i[\bar{\zeta}(\tau) \\
& \left.\times \dot{\zeta}(\tau)-\dot{\bar{\zeta}}(\tau) \zeta(\tau)] / 2-H_{S}(\bar{\zeta}, \zeta)\right\}, \\
S_{E}[\overline{\mathbf{z}}, \mathbf{z}]= & \sum_{k}-i \bar{z}_{k} z_{k}(t)+\int_{t_{0}}^{t} d \tau\left[i \bar{z}_{k} \dot{z}_{k}(\tau)\right. \\
& \left.-H_{E}(\overline{\mathbf{z}}, \mathbf{z})\right], \\
S_{I}[\overline{\mathbf{z}}, \mathbf{z}, \bar{\zeta}, \zeta]= & -\int_{t_{0}}^{t} d \tau H_{I}[\overline{\mathbf{z}}, \mathbf{z}, \bar{\zeta}, \zeta] .
\end{aligned}
$$

All the functional integrations are worked out over paths $\overline{\mathbf{z}}(\tau), \mathbf{z}(\tau), \bar{\zeta}(\tau)$, and $\zeta(\tau)$, the endpoints are $\overline{\mathbf{z}}(t) \equiv$ $\overline{\mathbf{z}}_{f}, \mathbf{z}\left(t_{0}\right) \equiv \mathbf{z}_{i}, \bar{\zeta}(t) \equiv \zeta_{f}$, and $\zeta\left(t_{0}\right) \equiv \zeta_{i}$.

Now we can calculate explicitly the influence functional of our model using the coherent state path-integral formalism. Substituting Eq. (10) into the actions of
Eq. A.2, we obtain the explicit form of the propagator. The path integral of the environmental part in the propagator can be exactly done by the stationary phase method [33, 34] with the boundary conditions $z_{k}\left(t_{0}\right)=z_{k i}$ and $\bar{z}_{k}(t)=\bar{z}_{k f}$. This method needs the equations of motion of the path,

$$
\dot{z}_{k}+i \Omega_{k} z_{k}=-i g_{k}^{*} \zeta, \dot{\bar{z}}_{k}-i \Omega_{k} \bar{z}_{k}=i g_{k} \bar{\zeta}
$$

where $\zeta$ and $\bar{\zeta}$ are treated as external sources. By formally integrating Eq. A.3 , we obtain A.3

$$
\begin{aligned}
& z_{k}(\tau)=z_{k i} e^{-i \Omega_{k} \tau}-i g_{k}^{*} \int_{0}^{\tau} d \tau^{\prime} e^{-i \Omega_{k}\left(\tau-\tau^{\prime}\right)} \zeta\left(\tau^{\prime}\right), \\
& \bar{z}_{k}(\tau)=\bar{z}_{k f} e^{i \Omega_{k}(\tau-t)}+i g_{k} \int_{\tau}^{t} d \tau^{\prime} e^{i \Omega_{k}\left(\tau-\tau^{\prime}\right)} \bar{\zeta}\left(\tau^{\prime}\right)
\end{aligned}
$$

By taking the reservoir to be initially at zero temperature (15), i.e., $\rho_{E}\left(\overline{\mathbf{z}}_{i}, \mathbf{z}_{i}^{\prime} ; 0\right)=1$, we finally can obtain Eq. (16) after substituting the result and Eq. (A.4) into Eq. (A.1.
[1] R. Alicki and K. Lendi, Quantum Dynamical Semigroups and Applications, Lecture Notes in physics, Vol. 717, 2nd ed. (Springer, Berlin, 2007)

[2] H.-P. Breuer and F. Petruccione, The Theory of Open Quantum Systems (Oxford University Press, Oxford, UK, 2002).

[3] U. Weiss, Quantum Dissipative Systems, 3rd ed. (World Scientific Press, Singapore, 2008)

[4] D. P. DiVincenzo, Nature 393, 113 (1998).

[5] E. Knill, R. Laflamme, and G. J. Milburn, Nature 409, 46 (2001).

[6] J. I. Cirac, A. K. Ekert, S. F. Huelga, and C. Macchiavello, Phys. Rev. A 59, 4249 (1999).

[7] D. P. DiVincenzo, Fortschr. Phys. 48, 771 (2000).

[8] J. I. Cirac, P. Zoller, H. J. Kimble, and H. Mabuchi, Phys. Rev. Lett. 78, 3221 (1997).

[9] L.-M. Duan, A. Kuzmich, and H. J. Kimble, Phys, Rev, A 67, 032305 (2003).

[10] C. W. Gardiner and P. Zoller, Quantum Noise (SpringerVerlag, Berlin, Germany, 2000).

[11] M. O. Scully and M. S. Zubairy, Quantum Optics (Cambridge University Press, Cambridge, UK, 1997).

[12] D. F. Walls and G. J. Milburn, Quantum Optics (Springer-Verlag, Berlin, 1994).

[13] H. J. Carmichael, An Open Systems Approach to Quantum Optics, Lecture Notes in Physics m18 (SpringerVerlag, Berlin, 1993).

[14] L. Mandel and E. Wolf, Optical Coherence and Quantum Optics (Cambridge University Press, England, 1995).

[15] M. Weissbluth, Photon-Atom Interactions (Academic Press, Boston, 1989).

[16] W. Vogel and D. G. Welsch, Lectures on Quantum Optics (Akademie Verlag, Berlin, 1994).

[17] G. Compagno, R. Passante, and F. Persico, Atom-Field Interactions and Dressed Atom (Cambridge University Press, Cambridge, 1995).

[18] A. O. Caldeira and A. J. Leggett, Ann. Phys. (N.Y.) 149,
374 (1983).

[19] A. J. Leggett, S. Chakravarty, A. T. Dorsey, M. P. A. Fisher, A. Garg, W. Zwerger, Rev. Mod. Phys. 59, 1 (1987).

[20] J. B. Majer, F. G. Paauw, A. C. J. ter Haar, C. J. P. M. Harmans, and J. E. Mooij, Phys. Rev. Lett. 94, 090501 (2005).

[21] A. J. Berkley, H. Xu, R. C. Ramos, M. A. Gubrud, F. W. Strauch, P. R. Johnson, J. R. Anderson, A. J. Dragt, C. J. Lobb, and F. C. Wellstood, Science 300, 1548 (2003).

[22] Y. A. Pashkin, T. Yamamoto, O. Astafiev, Y. Nakamura, D. V. Averin, and J. S. Tsai, Nature (London) 421, 823 (2003).

[23] B. Bellomo, R. Lo Franco, and G. Compagno, Phys. Rev. Lett. 99, 160502 (2007).

[24] M. A. Nielsen and I. L. Chuang, Quantum Computation and Quantum Information (Cambridge University Press, Cambridge, UK, 2000).

[25] A. Barenco, D. Deutsch, A. Ekert, and R. Jozsa, Phys. Rev. Lett. 74, 4083 (1995).

[26] M. J. Biercuk, H. Uys, A. P. VanDevender, N. Shiga, W. M. Itano, and J. J. Bollinger, Nature (London) 458, 996 (2009).

[27] S. Das and G S Agarwal, J. Phys. B 42205502 (2009).

[28] I. Sinaysky, F. Petruccione, and D. Burgarth, Phys. Rev. A 78, 062301 (2008).

[29] C. Anastopoulos and B. L. Hu, Phys. Rev. A 62, 033821 (2000).

[30] R. P. Feynman and F. L. Vernon, Ann. Phys. (N.Y.) 24, 118 (1963).

[31] A. O. Caldeira and A. J. Leggett, Physica A 121, 587 (1983).

[32] B. L. Hu, J. P. Paz, and Y. Zhang, Phys. Rev. D 45, 2843 (1992).

[33] W. M. Zhang, D. H. Feng, and R. Gilmore, Rev. Mod. Phys. 62, 867 (1990).

[34] J. R. Klauder, Phys. Rev. D 19, 2349 (1979). 
[35] R. Karrlein and H. Grabert, Phys. Rev. E 55, 153 (1997).

[36] F. Haake and R. Reibold, Phys. Rev. A 32, 2462 (1985).

[37] J.-H. An, Y. Yeo, and C. H. Oh, Ann. Phys. (NY) 324, 1737 (2009).

[38] J. H. An and W. M. Zhang, Phys. Rev. A 76, 042127 (2007).

[39] J. H. An, M. Feng, and W. M. Zhang, Quantum. Inf. Comput. 9, 0317 (2009).

[40] A. Lucke, C. H. Mak, and J. T. Stockburger J. Chem. Phys. 111, 10843 (1999).

[41] C.-H. Chou, T. Yu, and B. L. Hu, Phys. Rev. E 77, 011112 (2008).

[42] J. P. Paz and A. J. Roncaglia, Phys. Rev. Lett. 100, 220401 (2008).

[43] J. P. Paz and A. J. Roncaglia, Phys. Rev. A 79, 032102 (2009).

[44] M. W. Y. Tu and W. M. Zhang, Phys. Rev. B 78, 235311 (2008).

[45] M. W. Y. Tu, M. T. Lee, and W. M. Zhang, Quant. Info. Proc. 8, 631 (2009).

[46] J. S. Jin, M. T.W. Tu,W. M. Zhang, and Y. J. Yan, New J. Phys. 12, 083013 (2010).

[47] H. T. Tan and W. M. Zhang, Phys. Rev. A 83, 032102 (2011).

[48] C. U. Lei and W. M. Zhang, Ann. Phys. 327, 1408 (2012).

[49] W.-M. Zhang, P.-Y. Lo, H.-N. Xiong, M.W.-Y. Tu, and F. Nori, Phys. Rev. Lett. 109, 170402 (2012).

[50] S. Chaturvedi and F. Shibata, Z. Phys. B 35, 297 (1979).

[51] R. Shibata, Y. Takahashi and N. Hashitsume, J. Stat. Phys. 17171 (1977).

[52] G. A. Prataviera, A. C. Yoshida, and S. S. Mizrahi, Phys. Rev. A 87, 043831 (2013).

[53] S. Nakajima, Prog. Theor. Phys. 20, 948 (1958).

[54] R. Zwanzig, J. Chem. Phys. 33, 1338 (1960).

[55] J. Zhang, Y.-X. Liu, R.-B. Wu, K. Jacobs, and F. Nori, Phys. Rev. A 87, 032117 (2013).

[56] H.-P. Breuer, B. Kappler, and F. Petruccione, Phys. Rev. A 59, 1633 (1999).
[57] Y. J. Yan, Phys. Rev. A 58, 2721 (1998).

[58] E. Ferraro, M. Scala, R. Migliore, and A. Napoli, Phys. Rev. A 80, 042112 (2009).

[59] R. X. Xu and Y. J. Yan, J. Chem. Phys. 114, 3868 (2001).

[60] M. Schröer, U. Kleinekathöfer, and M. Schreiber, J. Chem. Phys. 124, 084903 (2006).

[61] K.-L. Liu and H.-S. Goan, Phys. Rev. A 76, 022312 (2007).

[62] P. Haikka and S. Maniscalco, Phys. Rev. A 81, 052103 (2010).

[63] P. Haikka, Phys. Scr. 2010, 014047 (2010).

[64] K. E. Cahill and R. J. Glauber, Phys. Rev. A 59, 1538 (1999).

[65] R. J. Glauber, Phys. Rev. 131, 2766 (1963).

[66] S. Shresta, C. Anastopoulos, A. Dragulescu, and B. L. Hu, Phys. Rev. A 71, 022109 (2005).

[67] A. Ghosh, S. S. Sinha, and D. S. Ray, Phys. Rev. E 86, 011138 (2012).

[68] A. Ishizaki and Y. Tanimura, Chem. Phys. 347, 185 (2008).

[69] L. D. Faddeev and A. A. Slavnov, Gauge Fields: Introduction to Quantum Theory (Benjamin-Cummings, Reading, MA, 1980).

[70] R. P. Feynman and A. R. Hibbs, Quantum Mechanics and Path Integrals (McGraw-Hill, New York, 1965).

[71] J.-G. Li, J. Zhou, and B. Shao, Phys. Rev. A 81, 062124 (2010).

[72] H. Z. Shen, M. Qin, and X. X. Yi, Phys. Rev. A 88, 033835 (2013).

[73] V. N. Shatokhin, S. Ya. Kilin, Opt. Commun. 174, 157 (2000).

[74] G. Lindblad, Commun. Math. Phys. 48, 119 (1976).

[75] V. Gorini, A. Kossakowski, and E. Sudarshan, J. Math. Phys. 17, 821 (1976).

[76] M. Thoss, H. Wang, and W. H. Miller, J. Chem. Phys. 115, 2991 (2001). 